\title{
UDP-Glucose 6-Dehydrogenase regulates hyaluronic acid production and promotes breast cancer progression
}

\author{
James M. Arnold ${ }^{1,2,3, \#}$, Franklin Gu1,2,3,\#, Chandrashekar R Ambati ${ }^{3}$, Uttam Rasaily ${ }^{2,3}$, \\ Esmeralda Ramirez-Pena ${ }^{4,5}$, Robiya Joseph ${ }^{4}$, Mohan Manikkam², Rebeca San Martin ${ }^{2}$, \\ Christy Charles ${ }^{1,2,3}$, Yinghong Pan ${ }^{6,7}$, Sujash S. Chatterjee ${ }^{6}$, Petra Den Hollander ${ }^{4}$, \\ Chandandeep Nagi ${ }^{8}$, Andrew G Sikora ${ }^{9}$, David Rowley ${ }^{2}$, Nagireddy Putluri ${ }^{2,3}$, \\ Balasubramanyam Karanam ${ }^{10}$, Sendurai A. Mani ${ }^{4}$, Arun Sreekumar ${ }^{*}, 1,2,3$ \\ ${ }^{1}$ Verna and Marrs McLean Department of Biochemistry and Molecular Biology, Baylor College of \\ Medicine, Houston, TX, 77030. \\ 2Department of Molecular and Cellular Biology, Baylor College of Medicine, Houston, TX, 77030, \\ USA. \\ ${ }^{3}$ Alkek Center for Molecular Discovery, Baylor College of Medicine, Houston, TX, 77030, USA. \\ ${ }^{4}$ Department of Translational Molecular Pathology, MD Anderson Cancer Center, Houston, TX, \\ 77030, USA. \\ ${ }^{5}$ Division of Cancer Prevention, National Cancer Institute, Rockville, MD, 20850, USA \\ ${ }^{6}$ Seq-N-Edit Core, Department of Biology and Biochemistry, University of Houston, Houston, TX, \\ 77004, USA. \\ ${ }^{7}$ UPMC Genome Center, Pittsburgh, PA, 15232, USA \\ ${ }^{8}$ Department of Pathology \& Immunology, Baylor College of Medicine, Houston, TX, 77030, USA. \\ ${ }^{9}$ Department of Otolaryngology-Head and Neck Surgery, Baylor College of Medicine, Houston, \\ TX, 77030, USA. \\ ${ }^{10}$ Department of Biology and Cancer Research, Tuskegee University, Tuskegee, AL 36088, USA.
}

\section{Abstract}

An improved understanding of the biochemical alterations that accompany tumor progression and metastasis is necessary to inform the next generation of diagnostic tools and targeted therapies.

Metabolic reprogramming is known to occur during the epithelial-mesenchymal transition (EMT), a process that promotes metastasis. Here, we identify metabolic enzymes involved in extracellular

\footnotetext{
Users may view, print, copy, and download text and data-mine the content in such documents, for the purposes of academic research, subject always to the full Conditions of use:http://www.nature.com/authors/editorial_policies/license.html\#terms

*Corresponding Author Arun Sreekumar, Ph.D., Professor, Department of Molecular and Cell Biology and, Verna and Marrs

McLean Department of Biochemistry and Molecular Biology, Baylor College of Medicine, Houston, TX, 77030, sreekuma@bcm.edu.

\#Authors contributed equally

The authors declare no potential conflicts of interest.

Competing Interests

The authors declare no potential competing financial interests in relation to the work described.
} 
matrix remodeling that are upregulated during EMT and are highly expressed in patients with aggressive mesenchymal-like breast cancer. Activation of EMT significantly increases production of hyaluronic acid which is enabled by the reprogramming of glucose metabolism. Using genetic and pharmacological approaches, we show that depletion of the hyaluronic acid precursor UDPglucuronic acid is sufficient to inhibit several mesenchymal-like properties including cellular invasion and colony formation in vitro, as well as tumor growth and metastasis in vivo. We found that depletion of UDP-glucuronic acid altered the expression of PPAR-gamma target genes and increased PPAR-gamma DNA binding activity. Taken together, our findings indicate that the disruption of EMT-induced metabolic reprogramming affects hyaluronic acid production as well as associated extracellular matrix remodeling and represents pharmacologically actionable target for the inhibition of aggressive mesenchymal-like breast cancer progression.

\section{Introduction}

Advancements in detection and early intervention have dramatically reduced breast cancer mortality rates (1). Decades of research into the biological processes that enable metastasis have yielded a simplified model known as the "metastatic cascade." The process known as the epithelial-mesenchymal transition (EMT) is believed to imbue epithelial breast cancer cells with mesenchymal-like properties (2). Cells having undergone EMT are more invasive and are capable of readily forming spheroids in three-dimensional culture, a process associated with increased metastatic potential of breast cancer cells (2). Our team and others have shown that EMT promotes significant metabolic reprogramming associated with poor patient outcomes (3). Leveraging our previous study with additional public gene expression datasets, here we identify a core set of metabolic enzymes upregulated during EMT and strongly expressed in breast cancer patients with mesenchymal-like gene expression signatures. Our results suggest that EMT induces significant production of the extracellular matrix component hyaluronic acid (HA). We hypothesized that increased HA synthesis necessitates reprogramming of the glucose metabolism, and that disrupting this metabolic reprogramming inhibits breast cancer invasion and mesenchymal-like properties. We demonstrate that EMT promotes reprogramming of glucose metabolism into HA precursor pathways and that genetic and pharmacological depletion of the HA precursor UDPglucuronic acid significantly reduces cellular invasion and colony formation in vitro, and tumor growth and metastasis in vivo, in mesenchymal-like breast cancer models.

\section{Results}

\section{Identification of Clinically Relevant Mesenchymal Metabolic Enzymes}

Our previous study of metabolic reprogramming induced by EMT identified several metabolic enzymes commonly regulated by three independent transcription factors-Twist, Snail, and Goosecoid—known to induce a mesenchymal-like phenotype (3). Here, we developed an integrative approach to use metabolomics data from these models with matched transcriptomics data in order to identify metabolic pathways that are transcriptionally altered during EMT. Leveraging the Kyoto Encyclopedia of Genes and Genomes (KEGG) database, we generated a list of enzymes known to interact with the significantly altered metabolites. The transcriptomics data was then filtered on this list, 
producing a set of 20 differentially regulated metabolic genes (Supplementary Figure 1A). Among the upregulated metabolic genes, UGDH was observed to be the most frequently altered in all breast cancer patients within the METABRIC cohort (4) (Supplementary Figure 1B).

To determine broader clinical relevance, we queried publicly available datasets $(4,5)$ containing mesenchymal-like breast cancer cell line models as well as patients with mesenchymal-like, claudin-low breast cancer to identify highly expressed metabolic genes (Figure 1A). We focused on metabolic genes at the union of all three analyses, which yielded 12 potential targets (Figure 1B). Pathway analysis of the upregulated metabolic genes indicated that the pathways associated with glucuronidation, amino sugars, and nucleotide sugars were highly represented (Figure 1C).

We tested each gene for associations with recurrence-free survival (RFS) (Table 1) in a cohort of estrogen receptor (ER)-negative breast cancers (6). UDP-Glucose Dehydrogenase (UGDH) yielded the largest significant hazard ratio with respect to RFS (Figure 1D). To further validate UGDH's clinical significance, immunohistochemistry was performed on a tissue microarray of breast cancer samples (Figure 1E). The TMA data revealed that UGDH staining was most intense in invasive malignant breast cancer samples, followed by metastatic breast cancer samples (Figure 1F). Lowest UGDH staining intensity was observed in non-malignant hyperplasia and benign tissue samples (Figure 1E).

Among the 12 metabolic genes identified in the overlap analysis (Figure 1B), both HAS2 and UGDH were directly related to the production of HA (Figure 1G). To validate our initial results and confirm that both UGDH and HAS2 were transcriptionally upregulated in the mesenchymal-like state, we measured the expression of both genes using RT-PCR in an epithelial cell line harboring an inducible Twist expression construct (2). Twist expression was induced by supplementing culture medium with $20 \mathrm{nM}$ 4-hydroxytamoxifen (OHT) for 14 days. The activation of Twist promoted a 7-fold increase in the expression of UGDH (Supplementary Figure 2B) and a 240-fold increase in the expression of HAS2 (Supplementary Figure 2C). Similarly, overexpression of Snail in HMLE cells induced a 4fold increase in UGDH expression (Supplementary Figure 2B). Furthermore, Snail induction of UGDH expression occurred in a manner that is dependent on FOXC2 expression (Supplementary Figure 2D). As a secreted product, HA synthesis can be quantified via competitive ELISA on spent cell culture media. Twist induction increased HA production 4.3-fold compared with control cells (Supplementary Figure 2E).

The glucosamine backbone of UDP-N-acetylglucosamine and the glucuronate backbone of UDP-glucuronic acid are both derived from glucose via the amino sugar pathway and the nucleotide sugar pathway, respectively (7) (Figure 2A). Therefore, we hypothesized that EMT requires metabolic reprogramming to produce the metabolic precursors for HA synthesis and the adoption of mesenchymal-like properties. The metabolic flux of glucose into HA precursors and other downstream fates was measured using universally labeled 13C-glucose. (Figures 2B-2E). Briefly, 13C labeled metabolites such as glucose and glutamine can be used to trace metabolic flux using mass spectrometry. Metabolite products downstream of the labeled precursor will be heavier by 1 neutron per labeled carbon, which 
are detected by the mass spectrometer as separate 'isotopomers'. Specifically, these are molecules that are chemically identical but differ by atomic mass, meaning they will elute from chromatography at the same time but produce distinct $\mathrm{m} / \mathrm{z}$ signatures. By convention, isotopomers containing only $12 \mathrm{C}$ are labeled ' $\mathrm{m}+0$ ' whereas isotopomers containing $n 13 \mathrm{C}$ atoms are labeled ' $m+n$ ' (e.g. universally labeled glucose is labeled ' $m+6$ '). Cells expressing Twist exhibited a 25\% increase in glucose turnover (Figure 2B), a $20 \%$ increased flux into UDP-glucuronic acid (Figure 2C), a 10\% increased flux into UDP-N-acetylglucosamine (Figure 2D), and no significant change in flux into citrate (Figure 2E). These results suggest that Twist expression and accompanying mesenchymal-like phenotype reprograms cellular metabolism to promote the utilization of glucose for biosynthetic purposes independent of existing bioenergetic demands.

\section{Genetic Depletion of UDP-Glucuronic Acid Inhibits Breast Cancer Invasion, Colony Formation, and Tumor Growth}

To examine the tumor-promoting function of UDP-glucuronic acid, shRNA knockdown (KD) of the enzyme UGDH was generated in the HMLE Twist-ER model, resulting in 90\% and $85 \%$ reduction in UGDH expression with two independent shRNA constructs (Figure 2F, Supplementary Figure 2F), respectively. To determine the effectiveness of UGDH KD, UDP-glucuronic acid was measured by steady-state mass spectrometry. In control cells, Twist induction produced a 3-fold increase in the pool size of UDP-glucuronic acid (Figure $2 \mathrm{G})$. Relative to control cells in the Twist-induced state, UGDH KD resulted in a 4-fold decrease in intracellular UDP-glucuronic acid in both KD models (Figure 2G). To test whether UDP-glucuronic acid was required to maintain mesenchymal-like properties, we assessed the cells using the transwell invasion assay and soft agar colony formation assay. UGDH KD in Twist-induced cells significantly decreased ( 25 fold) their invasive potential (Figures 2H, 2I), and these cells formed fewer colonies in soft agar (Supplementary Figures $2 \mathrm{G}, 2 \mathrm{H})$.

UGDH was also knocked down in the mesenchymal-like TNBC cell model MDA-MB-231 (M231). Using two independent shRNAs, $90 \%$ and $85 \% \mathrm{KD}$ of UGDH was achieved, assessed by western blot and RT-PCR (Figure 3A, Supplementary Figure 3A). To confirm pathway inhibition, intracellular UDP-glucuronic acid was measured using steady-state mass spectrometry. UGDH KD reduced intracellular UDP-glucuronic acid levels by nearly $50 \%$ in both KD models (Figure 3B). Next, to determine the influence of UDP-glucuronic acid levels on HA production, HA content in spent media for control and KD models was assessed by competitive ELISA. UGDH KD significantly decreased HA production by $60 \%$ and 50\%, respectively (Supplementary Figure 3B). Doubling time was assessed in vitro and no significant difference was found (Supplementary Figure 3C). To assess cellular invasion, the transwell assay was performed. UGDH KD resulted in a $60 \%$ and $55 \%$ decrease in invasive potential of MDA-MB-231 cells (Figure 3C, Supplementary Figure 3D). Cellular invasion was partially rescued by the supplementation of exogenous HA, restoring invasion of the KD models to $80 \%$ and $95 \%$ relative to the non-target (NT) control, respectively (Figure 3C, Supplementary Figure 3D). Additionally, the ability of UGDH KD cells to form colonies in soft agar was significantly inhibited. Relative to control, UGDH KD resulted in 
2.5-fold and 2.0-fold less in colonies formed over 14 days, respectively (Figure 3D, Supplementary Figure 3E).

Given that loss of UGDH and disruption of HA synthesis led to decreased invasion and colony formation without affecting proliferation, we hypothesized that cells lacking UGDH would be less metastatic in vivo. This hypothesis was tested using the chick chorioallantoic membrane (CAM) model (8). MDA-MB-231 cells constitutively expressing luciferase were seeded onto the CAM of a developing chicken embryo to assess tumor formation and invasion capabilities. 7 days after seeding, UGDH KD cells exhibited decreased cellular expansion in the CAM measured by luciferase activity (Supplementary Figure 3F). Additionally, UGDH KD cells exhibited significantly decreased invasion, measured by RTPCR of human-specific Alu sequence from extracted total genomic DNA (Supplementary Figure 3G). UGDH KD tumors produced significantly less HA in the extracellular space (Figure 3E), as measured by immunohistochemistry.

Next, xenograft tumors were generated via orthotopic injection of MDA-MB-231 UGDH KD or NT control cells into the right and left mammary fat pads (Supplementary Figure $3 \mathrm{H}$ ) of female SCID-Beige mice. UGDH KD models formed tumors that grew at a slower rate than the control cells, differences that were detected as early as 3 weeks post-injections (Figures 3F). In addition, pulmonary metastasis following tail vein injection was reduced in UGDH KD cells compared to the control (Figure 3G, Supplementary Figures 3I, 3J). Taken together, UGDH is required for tumor growth, metastasis, and HA production in vivo.

To assess the potential downstream impact of UGDH KD, RNA-seq was performed on the MDA-MB-231 control and KD cells (Figure 3H). Within the UGDH KD cells, several genes associated with lipid and fatty acid metabolism were altered, along with known targets of the PPAR signaling pathway (Figure 3I). Elevated expression of PPAR target genes, including FADS2, LIPG, and LPL, was confirmed via RT-PCR (Figure 3J), suggesting a potential relationship between UGDH and PPAR signaling. To test whether PPAR signaling involved PPAR-gamma, one of the known regulators of lipid metabolism, a PPAR-gamma DNA binding activity assay was performed. PPAR-gamma activity was significantly elevated in both UGDH KD models compared to NT control (Figure 3K). Taken together, this evidence implicates UGDH as a potential determinant of PPAR-gamma activity.

\section{4-Methylumbelliferone Inhibits Breast Cancer Invasion, Colony Formation, and Tumor Growth}

Studies have indicated that the pharmaceutical agent 4-methylumbelliferone (4-MU) is an effective inhibitor of HA synthesis (9). The mechanism of 4-MU inhibitor involves depleting intracellular UDP-glucuronic acid via UDP-glucuronosyltransferase (UGT)-mediated Phase II glucuronidation reactions (Supplementary Figure 4A) (10). Therefore, we hypothesized that pharmacological depletion of UDP-glucuronic acid via 4-MU would be sufficient to inhibit mesenchymal-like properties including invasion, colony formation, and metastasis. 4MU inhibited HA production in MDA-MB-231 cells at concentrations above $500 \mu \mathrm{M}$, with a calculated $\mathrm{IC}_{50}$ of $608 \mu \mathrm{M}$ (Supplementary Figure 4B). 4-MU did not affect on proliferation at concentrations less than $1 \mathrm{mM}$; however, cells treated with $5 \mathrm{mM}$ and $10 \mathrm{mM}$ grew 
significantly slower (Supplementary Figure 4C). We, therefore, carried out our experiments using $1 \mathrm{mM}$ 4-MU.

To confirm that 4-MU was depleting intracellular UDP-glucuronic acid, the metabolite was measured in cells 24 hours after drug treatment by mass spectrometry. We observed that treatment with $1 \mathrm{mM} 4-\mathrm{MU}$ led to a 55\% reduction in the intracellular pool of UDPglucuronic (Figure 4A). Treatment of MDA-MB-231 cells with $1 \mathrm{mM}$ 4-MU significantly reduced colony formation by 6 -fold, and cellular invasion by 55\%, compared to vehicletreated controls, with the latter getting rescued by addition of exogenous HA (Figures 4B-4E). To further evaluate the effects of 4-MU on breast cancer cells in vivo, xenograft tumors were generated via orthotopic injection of MDA-MB-231 cells into the left mammary fat pads of female SCID-Beige mice. Mice were randomized into two groups, with one group receiving $225 \mathrm{mg} / \mathrm{kg}$ of 4-MU via daily oral gavage and the other group receiving a vehicle control of $2 \%$ sucrose solution. The group receiving 4-MU exhibited significantly delayed tumor growth after 6 weeks (Figures 4F, 4G). Taken together, this suggests that pharmacological disruption of HA production via depletion of UDP-glucuronic acid, is an actionable target for inhibiting invasion, colony formation, and tumor growth.

Taken together, UGDH and its downstream product, UDP-glucuronic acid, is activated during EMT, contributes to extracellular matrix remodeling, and is required for invasion and tumor growth in vivo.

\section{Discussion}

Our study identified several metabolic alterations associated with EMT (Figure 1B, Supplementary Figure 1A), among which we focused on the clinically relevant metabolic enzyme UGDH, as its expression is most associated with low RFS in ER-negative breast cancer patients (Figure 1C). Moreover, the initial integrative analysis of the metabolomic and transcriptomic datasets suggests that amino sugar and nucleotide sugar pathways were transcriptionally upregulated during EMT (Supplementary Figure 1B). HAS2 also emerged as a candidate gene. HAS2 synthesizes HA from UDP-N-acetylglucosamine and UDPglucuronic acid, the end products of the amino sugar and nucleotide sugar pathways, respectively. Our data suggest that HA production is likely an important function of EMT. Previous studies in multiple cancer types have suggested that targeting UGDH and HA production may be sufficient to inhibit cancer invasion and metastasis (9,11-14). In this study, we aimed to strengthen the disparate connections between HA production, metabolic reprogramming, EMT, and breast cancer progression. Namely, EMT-induced HA production is a product of upstream metabolic reprogramming that is required for breast cancer progression. Moreover, our goal was to show that targeting the upstream determinants of HA production, specifically UGDH and UDP-glucuronic acid, were sufficient to inhibit pathological features of breast cancer.

To confirm our initial findings, we looked to an experimentally inducible model of EMT. Twist induction in the HMLE cell line led to significantly elevated HA production (Supplementary Figure 2E). Because HA is comprised of repeating sugar subunits derived from glucose (Figures 1F, 2A), we hypothesized that elevated HA production requires 
metabolic reprogramming. Indeed, overexpression of the EMT-activating transcription factors Snail and Twist induced UGDH expression (Supplementary Figure 2B) in a manner dependent on FOXC2, a transcription factor induced by EMT pathways (Supplementary Figure 2D). Furthermore, Twist induction increased glucose uptake (Figure 2B) and flux into the HA precursors UDP-glucuronic acid (Figure 2C) and UDP-N-acetylglucosamine (Figure 2D), but not citrate (Figure 2E). These findings are in agreement with earlier studies regarding glucose metabolism and EMT (15), and suggest that glucose reprogramming towards HA precursors is a feature of EMT. This is particularly interesting with respect to previous works revealing the importance of both the hexosamine biosynthetic pathway and the nucleotide sugar pathway to EMT and cancer progression $(11,16)$.

Next, as our preliminary data suggested UGDH is associated with poor RFS, we hypothesized that disruption of UGDH and its metabolic production UDP-glucuronic acid in mesenchymal-like cancer cells would inhibit HA production and mesenchymal-like properties. Using shRNAs, we found that KD of UGDH was sufficient to decrease invasion (Figures 2H, 2I) and colony formation (Supplementary Figures 2F, 2G), suggesting that these mesenchymal-like properties are dependent on UGDH. In agreement, KD of UGDH resulted in the loss of HA production (Supplementary Figure 3B), invasion (Figure 3C, Supplementary Figure 3D), and colony formation (Figure 3D, Supplementary Figure 3E) in the mesenchymal-like breast cancer model, MDA-MB-231. Supplementation with exogenous HA was sufficient to rescue the invasive phenotype (Figure 3C, Supplementary Figure 3D). Furthermore, KD of UGDH in vivo significantly reduced localized HA production (Figure 3E) and tumor growth (Figures 3F). In addition, pulmonary metastasis was reduced in UGDH KD cells (shU1) relative to control NT cells following tail vein injection (Figure 3G) with the presence of metastatic luciferase-labeled M231 cells (Supplementary Figure 3I) confirmed by H\&E staining (Supplementary Figure 3J), suggesting that the availability of UDP-glucuronic acid may be critical to the establishment of a pro-tumorigenic microenvironment.

To gain insights into the intracellular effects of UGDH KD, we performed RNA-seq on the MDA-MB-231 KD and NT control cells. The results suggested loss of UGDH promoted several genes associated with lipid and fatty acid metabolism and fell under the PPAR signaling pathway (Figure 3J). We confirmed these genes were upregulated in the KD cells (Figure 3K) and found that PPAR-gamma had higher DNA binding activity in the KD models relative to NT control (Figure 3I). It is unclear what connects UGDH expression to PPAR-gamma activity, although our current hypothesis is that loss of intracellular UDPglucuronic acid may cause accumulation of xenobiotic compounds normally cleared by Phase II glucuronidation reactions, some of which may be acting as endogenous PPAR activators. Further experiments are warranted to elucidate the mechanism underlying this phenomenon.

The effects of UGDH KD were phenocopied with the treatment of the HA inhibitor 4-MU, which is known to act via the depletion of intracellular UDP-glucuronic acid (Supplementary Figure 4A, Figure 4A). Treatment with 4-MU significantly inhibited HA production (Supplementary Figure 4B), colony formation (Figures 4B, 4C), and invasion (Figures 4D, 4E) in vitro and was rescued in part by the supplementation of exogenous HA 
(Figures 4D, 4E). Taken together, these data suggest that a strategy to deplete intracellular UDP-glucuronic acid may be beneficial to inhibiting tumor growth and invasion.

HA production by both cancer and stromal cells have been implicated in tumor progression in several cancer types, mediated in part by interactions between HA and HA receptors such as CD44 and RHAMM $(12,17)$. Beyond its known role in EMT, the HA-CD44 pathway has also been linked to resistance to radiation and chemotherapy in cancer cells. The presence of HA in tumors is sufficient to inhibit the formation of intratumoral vasculature and increase the expression of drug transporters to increase chemoresistance $(18,19)$. Furthermore, HA depletion in mouse models of pancreatic cancer increased sensitivity to chemotherapy (19). Taken in combination with our study showing that HA synthesis is involved in tumor growth and metastasis, this suggests that targeting HA production through UGDH can simultaneously inhibit multiple cancer pathologies.

In conclusion, this study demonstrates that the EMT promotes metabolic reprogramming to increase glucose flux into HA via amino sugar and nucleotide sugar pathways. Depleting UDP-glucuronic acid via UGDH KD or pharmacological inhibition significantly inhibits mesenchymal-like properties including invasion, colony formation, and tumor growth in an HA-dependent manner and may be an actionable molecular target to inhibit aggressive mesenchymal-like breast cancer progression.

\section{Materials and Methods}

\section{Cell Culture}

All cell lines were verified using STR (Short Tandem Repeat) DNA fingerprinting at MD Anderson Cancer Center and were tested negative for mycoplasma contamination using MycoAlert Detection Kit (Lonza, Cat \# LT07-418).

Immortalized human mammary epithelial (HMLE) cells harboring a tamoxifen-inducible Twist expression construct were obtained from Dr. Sendurai Mani. Cells were cultured as previously reported $(2,20,21)$. Twist expression was induced by supplementing media with 20 nM 4-hydroxytamoxifen (OHT) (Sigma, Cat \# H7904-5MG).

MDA-MB-231 cells were obtained from the BCM Advanced Technology Core shared resource. Cells were cultured in DMEM media (Corning, Cat \# 10-013-CV) supplemented with $10 \%$ fetal bovine serum (Gibco, Cat \# 10438034). Stable UGDH knockdown (KD) cells were produced using GIPZ lentiviral plasmids carrying shRNA specific to UGDH, or non-target control. GIPZ plasmids were obtained from the Cell-Based Assay Screening Service (CBASS) core at Baylor College of Medicine. Two independent shRNA clones targeting UGDH were used for transduction at a viral titer of 5 MOI. Cells with stable KD of the gene were cultured in respective media supplemented with $1 \mu \mathrm{g} / \mathrm{ml}$ of Puromycin (Sigma, Cat \# P8833-10MG). Cells were further transduced with a constitutive luciferase expression lentiviral vector (GenTarget, Cat \# LVP402-PBS) for use in in vivo models. 
4-methylumbelliferone (4-MU) (Sigma Cat \# M1381) was resuspended in media at defined concentrations as indicated in the results section, or vehicle control (DMSO, Fisher, Cat \# BP231).

\section{Quantitative PCR}

Standard RNA extraction (RNeasy Mini Kit from QIAGEN), reverse transcription (Quanta Biosciences, Cat \# 95048-500) and SYBR green (Life Technologies, Cat \# 4385614) realtime PCR (RT-PCR) were performed (26). Primers used in this study are listed in Table 2.

Western blot antibodies-UGDH protein levels were measured using UGDH-specific antibody (Origene, TA810531).

\section{UGDH Tissue Microarray}

Tissue microarray containing of 206 breast cancer samples (Cat \#BR2082a) was purchased from US Biomax, Inc (Rockville, MD). Comparisons were made between the following groups: "Non-Malignant", comprised of 16 cases of inflamed breast, 16 cancer adjacent normal breast tissues, 8 cases of fibroadenoma, and 16 cases of adenosis with hyperplasia of the breast; "Invasive", comprised of 68 cases of invasive ductal carcinoma, 22 cases of lobular carcinoma, 4 cases of squamous cell carcinoma, 23 cases of intraductal carcinoma, and 3 cases of lobular carcinoma in situ; and "Metastatic", comprised of 32 cases of metastatic carcinoma breast tissues. Staining was scored on 0-3 scale by a breast pathologist in a blinded manner, and scores were plotted in R (27). Details of immunohistochemistry protocols are available in Supplementary Materials and Methods.

\section{Metabolomics}

Samples were prepared and analyzed for mass spectrometry-based metabolomics using Multiple Reaction Monitoring (MRM) as previously reported $(3,28,29)$. Briefly, frozen cell pellets stored at $-80^{\circ} \mathrm{C}$ were thawed at $4{ }^{\circ} \mathrm{C}$ and subjected to three freeze-thaw cycles in liquid nitrogen before the addition of $750 \mu \mathrm{L}$ of ice-cold methanol:water (4:1) containing 20 $\mu \mathrm{L}$ of spiked internal standard to each cell extract. Ice cold chloroform and then water were sequentially added in a 3:1 ratio to each cell extract. Organic and aqueous layers were isolated and then combined before de-proteinization using a $3 \mathrm{kDa}$ Amicon Ultracel molecular filter (Millipore, Billerica, MA). Filtrates were dried in vacuum (Genevac EZ-2plus, Gardinier, NY) and then resuspended in 1:1 methanol:water with $0.1 \%$ formic acid for mass spectrometry.

\section{Steady-state Metabolomic Analysis}

To quantify intracellular UDP-glucuronic acid, the following transitions were monitored in negative mode: precursor ion 579.02; product ions 402.9, 323.1, 78.9. The method was generated using a standard reference (Sigma, Cat \# U6751-100MG).

\section{Metabolic Flux Analysis}

To quantify metabolc flux of glucose, 4 replicates of 5 million +/- OHT treated HMLE Twist-ER cells were plated and grew overnight in normal media. Cells were labeled with 
U- ${ }^{13}$ C-glucose (Cambridge Isotope Laboratories, Cat \# CLM-1396). After 6 hours, cells were washed thrice with ice cold PBS, scraped, and immediately flash frozen in liquid nitrogen and stored at $-80^{\circ} \mathrm{C}$ until metabolite extraction was performed. Metabolites were extracted as previously reported (29). The transitions listed in Table 3 were used to quantify the distribution of labeled carbon from glucose into the intracellular pools of glucose, UDPglucuronic acid, UDP-N-acetylglucosamine, and citrate.

\section{Transwell Invasion Assay}

Cellular invasion was quantified using the transwell invasion assay as previously reported (30). Briefly, Matrigel-coated transwell chambers with $8.0 \mu \mathrm{m}$ PET membranes (Corning, Cat\# 354483) were rehydrated with serum-free media before being seeded with 10,000 cells per chamber. Cells were then allowed to invade towards media containing serum for 48 hours. For hyaluronic acid rescue studies, sodium hyaluronate (Lifecore Biomedical, Cat \# HA15M-1) was resuspended in media at $4 \mathrm{mg} / \mathrm{ml}$. Experimental samples were performed in triplicate and pooled.

Colony formation-Colony formation was assessed as previously reported (31). Colonies with diameter greater than $80 \mu \mathrm{m}$ were quantified and tabulated in Excel. Triplicate experiments were performed per condition and pooled data was used for analysis.

\section{Chorioallantoic Membrane Assay}

Fertilized White Leghorn Chicken eggs were prepared for cancer cell inoculation as previously published (8). Each egg was inoculated with 2 million cells per condition. 6 eggs were run per condition and repeated in three independent experiments.

For in vivo imaging, $100 \mu 115 \mathrm{mg} / \mathrm{ml}$ luciferase substrate was pippetted directly on the tumor and immediately imaged. Images were collected on a Xenogen IVIS 200 station using Living Image 4.2 software from PerkinElmer (Waltham, MA), set to an exposure time of 1 second with F/stop set to $1 / 2$ and medium binning. Analysis of the data was performed in Microsoft Excel. Significance was calculated using Student's unpaired two-tailed t-test.

Tissues from the lower CAM were dissected, collected, flash-frozen. Frozen samples were pulverized, and genomic DNA extracted following the protocol described in the DNeasy Blood and Tissue kit from Qiagen (Germantown, MD). The invaded human cells in the chicken cell background were quantified by performing quantitative RT-PCR for humanspecific Alu sequence (Table 4), as previously reported (32).

Hyaluronic acid density and UGDH expression were assessed in the tumor and surrounding environment of the collected upper CAM samples using immunohistochemistry.

In vivo studies-Mice were housed in pathogen-free cages containing 4 mice total and randomized into treatment groups based on cage. Sample sizes for animal studies were determined based on previously reported studies and experiments from our laboratory. Mouse studies were not blinded and no data was excluded. All animal work was done in accordance with a protocol approved by the Baylor College of Medicine Institutional Animal Care and Use Committee. 


\section{Metastasis Assay}

$100 \mu \mathrm{l}$ of $5 \times 10^{5}$ luciferase labeled cells were injected into the lateral tail vein of 6-week-old female SCID-Beige mice purchased from Charles River (Wilmington, MA). Each week following tail vein injection anesthetized mice were given retro-orbital injections of $100 \mu \mathrm{l}$ of $10 \mathrm{mg} / \mathrm{ml}$ luciferase substrate (GoldBio, Cat \# LUCK-1G) and imaged immediately. Images were collected on a Xenogen IVIS 200 station using Living Image 4.2 software (PerkinElmer), set to an exposure time of 1 second with F/stop set to $1 / 2$ and medium binning.

\section{Mouse Xenograft Tumors}

UGDH Knockdown-Xenograft tumors were generated by injecting 200,000 luciferaselabeled MDA-MB-231 control (shNT) or UGDH knockdown (shU1) cells into the left and right mammary fat pads of 6-week-old female SCID-Beige mice purchased from Charles River (Wilmington, MA). Tumor length (L) and width (W) was measured weekly using calipers. Tumor volume was calculated using the formula $\mathrm{L} \times \mathrm{W}^{2} 0.52$.

4-Methylumbelliferone-Xenograft tumors were generated via orthotopic injection of M231 cells into the left mammary fat pads of female SCID-Beige mice purchased from Charles River (Wilmington, MA). Mice were randomized into two groups-one group received $225 \mathrm{mg} / \mathrm{kg}$ of 4-MU (Sigma, Cat \# M1508-10G) resuspended in 2\% sucrose via daily oral gavage, and one group receiving a vehicle control of $2 \%$ sucrose solution. Tumor length (L) and width (W) was measured weekly using calipers for 6 weeks. Tumor volume was calculated using the formul1a $\mathrm{L} \times \mathrm{W}^{2} \times 0.52$.

\section{RNA-Seq Analysis}

Briefly, total RNA was extracted from biological triplicates of shNT, shU1, and shU2 M231 cells with the Qiagen RNeasy kit (Qiagen, Cat \# 74104). Libraries were prepared with Ovation ${ }^{\circledR}$ Universal Plus mRNA-Seq (NuGen, Cat \# 0508-32) using 200 ng input RNA. Samples were sequenced on an Illumina NextSeq 500 for 150 cycles yielding 130M 76PE reads. Data was analyzed using the RNA Express pipeline on BaseSpace (basespace.illumina.com). Alignment was performed with STAR aligner to UCSC hg19 human genome. Counts and differential genes were produced using DESeq2. Raw data and processed results can be accessed online under GEO accession number GSE118772.

Significantly differential genes were defined as possessing an FDR-corrected p-value $<0.05$ and a log-fold change $>1$ (upregulated) or $<-1$ (downregulated). Pathway analysis was performed on significantly upregulated and significantly downregulated gene sets independently using the Enrichr platform (33).

\section{Statistical analysis}

Unless otherwise stated, all samples were assayed in triplicate. All in vitro experiments were repeated at least three independent times. Unless otherwise indicated, data are represented as mean \pm standard error of the mean (SEM), and significance was calculated using Student's unpaired two-tailed t-test. 


\section{Code availability}

The source code used is available on https://github.com/SreekumarLab/Publications.

\section{Supplementary Material}

Refer to Web version on PubMed Central for supplementary material.

\section{Acknowledgements}

Thanks to Mariana Villanueva MS and Ravi Pathak Ph.D. M.B.A, in the Baylor College of Medicine Advanced in vivo Models Core for assistance with chicken chorioallantoic membrane assays. This research was partially funded by National Cancer Institute grant numbers U01CA179674-01A1 (AS), National Science Foundation NSF PHY-1605817 (SAM), P30CA125123 Metabolomics Shared Resources (AS), R01CA220297 (NP), and partially supported by P50CA186784 to C.K. Osborne; CPRIT grant numbers RP17005 to the Proteomics and Metabolomics Core Facility (AS and NP); CPRIT Core Facilities Support Grant RP170691 (ML and AS); funds from the Alkek Center for Molecular Discovery (AS) and Agilent Technologies Center for Excellence in Mass Spectrometry (AS); UH Division of Research, UH College of Natural Sciences and Mathematics, and Department of Biology \& Biochemistry, NRUF MINOR CORE 17 Grant to support Seq-N-Edit Core.

\section{References}

1. Siegel RL, Miller KD, Jemal A. Cancer Statistics, 2017. CA Cancer J Clin. 2017 1;67(1):7-30. [PubMed: 28055103]

2. Mani SA, Guo W, Liao M-J, Eaton EN, Ayyanan A, Zhou AY, et al. The epithelial-mesenchymal transition generates cells with properties of stem cells. Cell. 20085 16;133(4):704-15. [PubMed: 18485877]

3. Bhowmik SK, Ramirez-Peña E, Arnold JM, Putluri V, Sphyris N, Michailidis G, et al. EMT-induced metabolite signature identifies poor clinical outcome. Oncotarget. 201512 15;6(40):42651-60. [PubMed: 26315396]

4. Curtis C, Shah SP, Chin S-F, Turashvili G, Rueda OM, Dunning MJ, et al. The genomic and transcriptomic architecture of 2,000 breast tumours reveals novel subgroups. Nature. 20124 18;486(7403):346-52. [PubMed: 22522925]

5. Neve RM, Chin K, Fridlyand J, Yeh J, Baehner FL, Fevr T, et al. A collection of breast cancer cell lines for the study of functionally distinct cancer subtypes. Cancer Cell. 2006 12;10(6):515-27. [PubMed: 17157791]

6. Györffy B, Lanczky A, Eklund AC, Denkert C, Budczies J, Li Q, et al. An online survival analysis tool to rapidly assess the effect of 22,277 genes on breast cancer prognosis using microarray data of 1,809 patients. Breast Cancer Res Treat. 2010 10;123(3):725-31. [PubMed: 20020197]

7. Vigetti D, Viola M, Karousou E, De Luca G, Passi A. Metabolic control of hyaluronan synthases. Matrix Biol. 2014 4;35:8-13. [PubMed: 24134926]

8. Li M, Pathak RR, Lopez-Rivera E, Friedman SL, Aguirre-Ghiso JA, Sikora AG. The In Ovo Chick Chorioallantoic Membrane (CAM) Assay as an Efficient Xenograft Model of Hepatocellular Carcinoma. J Vis Exp. 2015 10 9;(104).

9. Yates TJ, Lopez LE, Lokeshwar SD, Ortiz N, Kallifatidis G, Jordan A, et al. Dietary supplement 4methylumbelliferone: an effective chemopreventive and therapeutic agent for prostate cancer. J Natl Cancer Inst. 2015 7;107(7).

10. Kultti A, Pasonen-Seppänen S, Jauhiainen M, Rilla KJ, Kärnä R, Pyöriä E, et al. 4Methylumbelliferone inhibits hyaluronan synthesis by depletion of cellular UDP-glucuronic acid and downregulation of hyaluronan synthase 2 and 3. Exp Cell Res. 20097 1;315(11):1914-23. [PubMed: 19285976]

11. Oyinlade O, Wei S, Lal B, Laterra J, Zhu H, Goodwin CR, et al. Targeting UDP-a-d-glucose 6dehydrogenase inhibits glioblastoma growth and migration. Oncogene. 2018 5;37(20):2615-29. [PubMed: 29479058] 
12. Misra S, Hascall VC, Markwald RR, Ghatak S. Interactions between Hyaluronan and Its Receptors (CD44, RHAMM) Regulate the Activities of Inflammation and Cancer. Front Immunol. 2015;6:201. [PubMed: 25999946]

13. Saito T, Dai T, Asano R. The hyaluronan synthesis inhibitor 4-methylumbelliferone exhibits antitumor effects against mesenchymal-like canine mammary tumor cells. Oncol Lett. 2013 3;5(3): 1068-74. [PubMed: 23426189]

14. Sato N, Kohi S, Hirata K, Goggins M. Role of hyaluronan in pancreatic cancer biology and therapy: Once again in the spotlight. Cancer Sci. 2016 5;107(5):569-75. [PubMed: 26918382]

15. Dong C, Yuan T, Wu Y, Wang Y, Fan TWM, Miriyala S, et al. Loss of FBP1 by Snail-mediated repression provides metabolic advantages in basal-like breast cancer. Cancer Cell. 20133 18;23(3):316-31. [PubMed: 23453623]

16. Lucena MC, Carvalho-Cruz P, Donadio JL, Oliveira IA, de Queiroz RM, Marinho-Carvalho MM, et al. Epithelial Mesenchymal Transition Induces Aberrant Glycosylation through Hexosamine Biosynthetic Pathway Activation. Journal of Biological Chemistry. 20166 17;291(25):12917-29. [PubMed: 27129262]

17. Toole BP. Hyaluronan-CD44 Interactions in Cancer: Paradoxes and Possibilities. Clinical Cancer Research. 200912 15;15(24):7462-8. [PubMed: 20008845]

18. Ricciardelli C, Ween MP, Lokman NA, Tan IA, Pyragius CE, Oehler MK. Chemotherapy-induced hyaluronan production: a novel chemoresistance mechanism in ovarian cancer. BMC Cancer. 2013 12;13(1):476. [PubMed: 24124770]

19. Jacobetz MA, Chan DS, Neesse A, Bapiro TE, Cook N, Frese KK, et al. Hyaluronan impairs vascular function and drug delivery in a mouse model of pancreatic cancer. Gut. 2013 1;62(1): 112-20. [PubMed: 22466618]

20. Hollier BG, Tinnirello AA, Werden SJ, Evans KW, Taube JH, Sarkar TR, et al. FOXC2 expression links epithelial-mesenchymal transition and stem cell properties in breast cancer. Cancer Res. 2013 3 15;73(6):1981-92. [PubMed: 23378344]

21. Battula VL, Evans KW, Hollier BG, Shi Y, Marini FC, Ayyanan A, et al. Epithelial-mesenchymal transition-derived cells exhibit multilineage differentiation potential similar to mesenchymal stem cells. Stem Cells. 2010 8;28(8):1435-45. [PubMed: 20572012]

22. Taube JH, Herschkowitz JI, Komurov K, Zhou AY, Gupta S, Yang J, et al. Core epithelial-tomesenchymal transition interactome gene-expression signature is associated with claudin-low and metaplastic breast cancer subtypes. Proc Natl Acad Sci USA. 20108 31;107(35):15449-54. [PubMed: 20713713]

23. Ritchie ME, Phipson B, Wu D, Hu Y, Law CW, Shi W, et al. limma powers differential expression analyses for RNA-sequencing and microarray studies. Nucleic Acids Research. 20154 20;43(7):e47-e47. [PubMed: 25605792]

24. Possemato R, Marks KM, Shaul YD, Pacold ME, Kim D, Birsoy K, et al. Functional genomics reveal that the serine synthesis pathway is essential in breast cancer. Nature. 20118 18;476(7360): 346-50. [PubMed: 21760589]

25. Lehmann BD, Bauer JA, Chen X, Sanders ME, Chakravarthy AB, Shyr Y, et al. Identification of human triple-negative breast cancer subtypes and preclinical models for selection of targeted therapies. Journal of Clinical Investigation. 20117 1;121(7):2750-67. [PubMed: 21633166]

26. Putluri N, Shojaie A, Vasu VT, Vareed SK, Nalluri S, Putluri V, et al. Metabolomic profiling reveals potential markers and bioprocesses altered in bladder cancer progression. Cancer Res. 2011 12 15;71(24):7376-86. [PubMed: 21990318]

27. Gentleman R Bioinformatics and computational biology solutions using R and Bioconductor [Internet]. New York: Springer Science+Business Media; 2005 [cited 2019 Apr 15]. Available from: 10.1007/0-387-29362-0

28. Putluri N, Maity S, Kommagani R, Kommangani R, Creighton CJ, Putluri V, et al. Pathway-centric integrative analysis identifies RRM2 as a prognostic marker in breast cancer associated with poor survival and tamoxifen resistance. Neoplasia. 2014 5;16(5):390-402. [PubMed: 25016594]

29. Dasgupta S, Putluri N, Long W, Zhang B, Wang J, Kaushik AK, et al. Coactivator SRC-2dependent metabolic reprogramming mediates prostate cancer survival and metastasis. Journal of Clinical Investigation. 20153 2;125(3):1174-88. [PubMed: 25664849] 
30. Marshall J Transwell(®) invasion assays. Methods Mol Biol. 2011;769:97-110. [PubMed: 21748672]

31. Werden SJ, Sphyris N, Sarkar TR, Paranjape AN, LaBaff AM, Taube JH, et al. Phosphorylation of serine 367 of FOXC2 by p38 regulates ZEB1 and breast cancer metastasis, without impacting primary tumor growth. Oncogene. 2016 17;35(46):5977-88. [PubMed: 27292262]

32. van der Horst EH, Leupold JH, Schubbert R, Ullrich A, Allgayer H. TaqMan-based quantification of invasive cells in the chick embryo metastasis assay. BioTechniques. 2004 12;37(6):940-2, 944, 946. [PubMed: 15597543]

33. Kuleshov MV, Jones MR, Rouillard AD, Fernandez NF, Duan Q, Wang Z, et al. Enrichr: a comprehensive gene set enrichment analysis web server 2016 update. Nucleic Acids Res. 2016 08;44(W1):W90-97. [PubMed: 27141961] 
A

$\mathrm{D}$

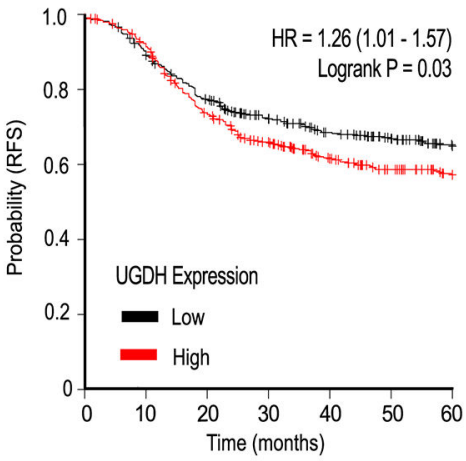

$\mathrm{F}$

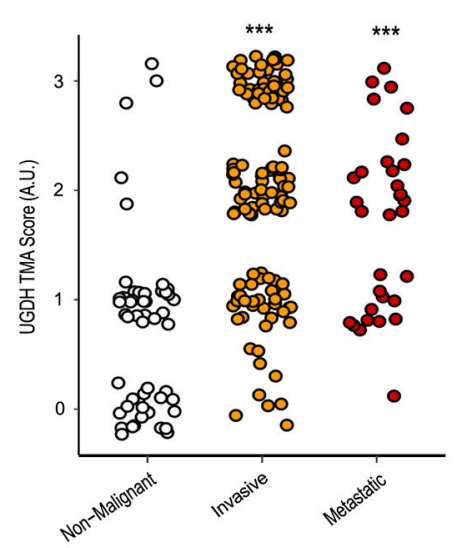

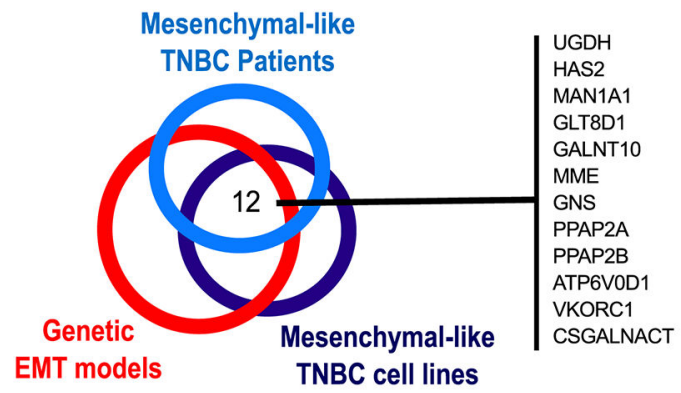

C

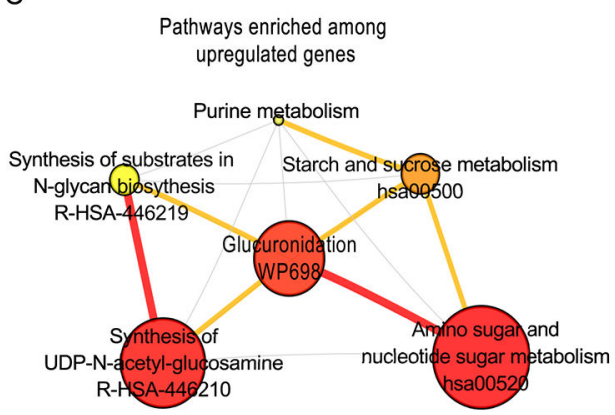

E

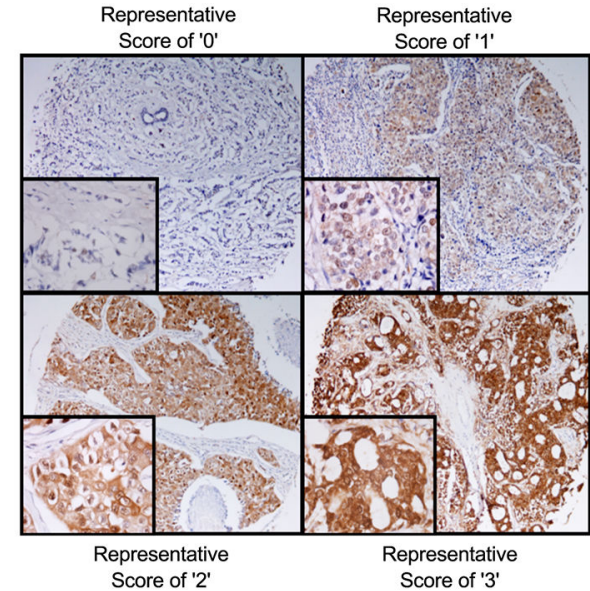

G

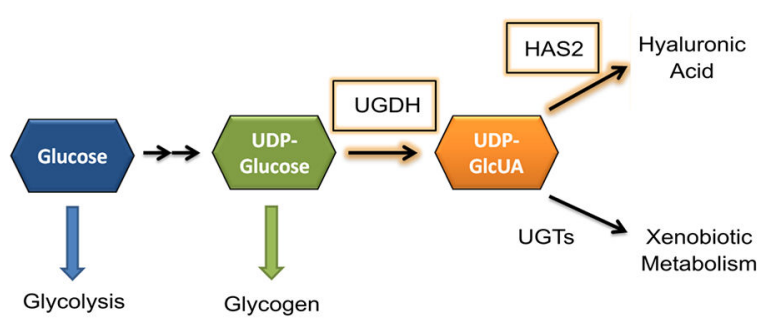

Figure 1: Identification of UGDH as a clinically relevant metabolic enzyme associated with mesenchymal-like gene expression and cancer progression.

A) Integrated gene expression analysis of breast cancer patient data, intrinsic cell lines, and in vitro models of EMT revealed 12 metabolic enzymes and transporters which are significantly associated with a mesenchymal-like gene signature. B) List of 12 metabolic genes upregulated in EMT models. C) Network visualization of metabolic pathways enriched among metabolic genes upregulated in EMT-TF models relative to control. Node size is proportional to pathway enrichment score, and node color is proportional to FDRadjusted p-value. Edge size and color is proportional to the number of shared genes between 
two nodes. D) Elevated expression of Uridine Diphosphate Glucose Dehydrogenase (UGDH) expression is associated with decreased recurrence-free survival (RFS) among a cohort of ER-negative patients. E) Representative images of UGDH staining within the TMA. A score of " 0 " indicates the absence of UGDH staining whereas a score of "3" indicates intense UGDH staining. F) TMA scores grouped by breast cancer type shows UGDH is significantly elevated in invasive and metastatic forms of breast cancer compared to non-malignant samples. See Methods section for detailed group definitions. G) A simplified model of the metabolic context of UGDH and its direct product, uridine diphosphate glucuronic acid (UDP-GlcUA). Briefly, carbon from glucose is converted into uridine diphosphate glucose (UDP-glucose) via a series of metabolic reactions. The enzyme UGDH converts UDP-glucose to UDP-GlcUA. UDP-GlcUA is used as the substrate for glucuronidation reactions by UDP-glucuronosyltransferases (UGTs), which is important in clearance of xenobiotic metabolites. Additionally, UDP-GlcUA is used as a precursor for glycosylaminoglycans including Dermatan sulfate, Chondroitin sulfate, and hyaluronic acid. Hyaluronic acid is synthesized from hyaluronic acid synthases, including HAS2, which was identified along with UGDH as one of the 12 enzymes associated with mesenchymal-like gene expression. Together, this suggests that mesenchymal-like cells will increase UDPglucuronic acid production and hyaluronic acid synthesis. 


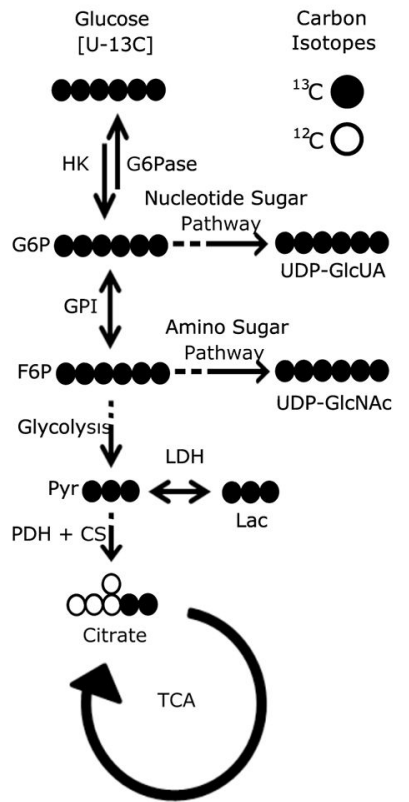

F

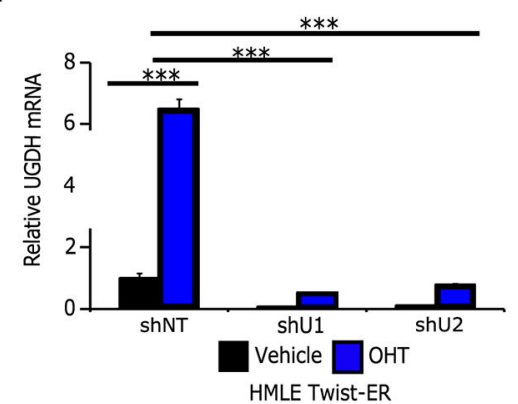

$\mathrm{H}$

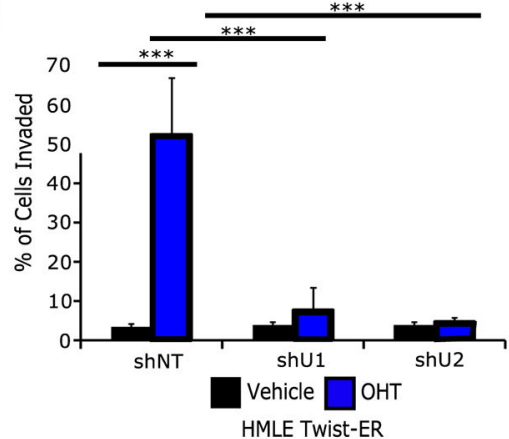

B

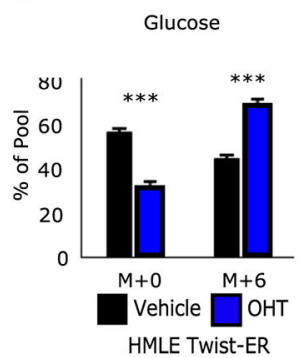

D

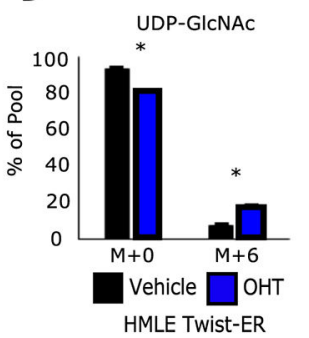

C

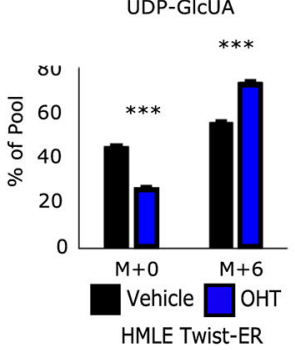

E

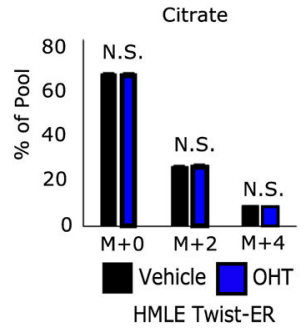

G

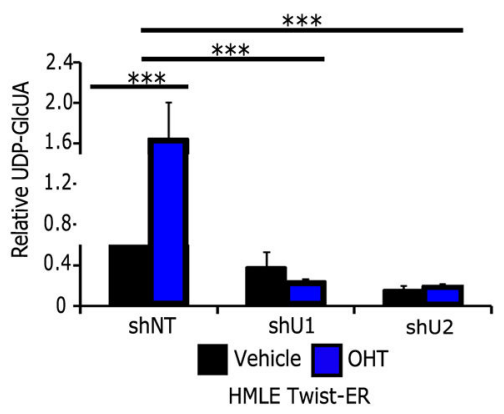

I

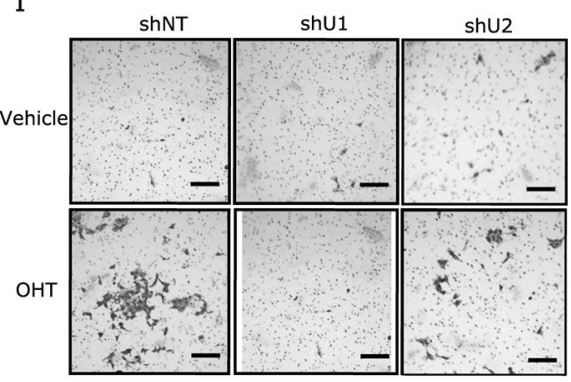

Figure 2: Twist promotes a mesenchymal-like phenotype which reprograms glucose metabolism to favor HA production.

A) Simplified carbon fate map from glucose to the HA precursors UDP-glucuronic acid (UDP-GlcUA) and UDP-N-acetylglucosamine (UDP-GlcNAc), as well as towards energy metabolism via glycolysis and entry into the tricarboxylic acid (TCA) pathway via citrate. A ${ }^{13} \mathrm{C}$ universally-labeled glucose moiety will contribute 6 carbons to both UDP-GlcUA and UDP-GlcNAc, and two carbons to citrate on the first turn of the TCA. Citrate may obtain up to four labeled carbons upon a second turn of the TCA. After 6 hours of incubation with ${ }^{13} \mathrm{C}$ universally-labeled glucose OHT-treated cells possessed: B) a $25 \%$ increase in the 
proportion of labeled glucose, C) a $20 \%$ increase in the proportion of labeled UDP-GlcUA, D) a $10 \%$ increase in the proportion of labeled UDP-GlcNAc, and E) no change in labeled citrate. F) shRNA-mediated knockdown (KD) of UGDH in control and OHT-treated HMLE Twist-ER cells. G) UGDH KD results in significantly decreases intracellular UDP-GlcUA. H) UGDH KD significantly decreases cellular invasion. I) Representative images of invaded cells. Scale bars $=100 \mu \mathrm{m}$. N.S. $=$ not significant, $* \mathrm{p}<0.05, * * *<0.001$, versus control. 
A
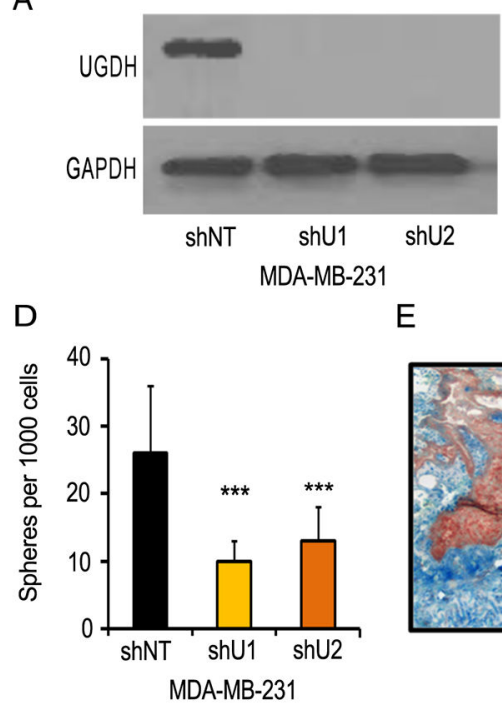

G

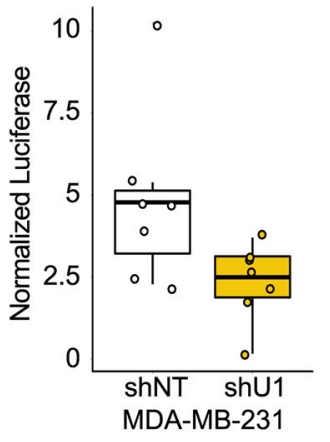

I

Concepts Associated with Upregulated Genes in UGDH Knockdown cells

Pathways in cance

PPAR signaling pathway

Glycerolipid metabolism

Glycosphingolipid biosynthesis - ganglio series

Fatty acid metabolism

Biosynthesis of unsaturated fatty acids
E

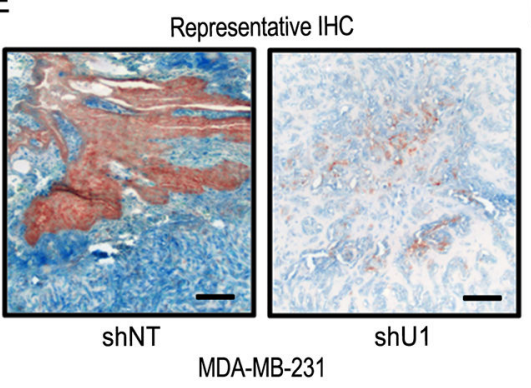

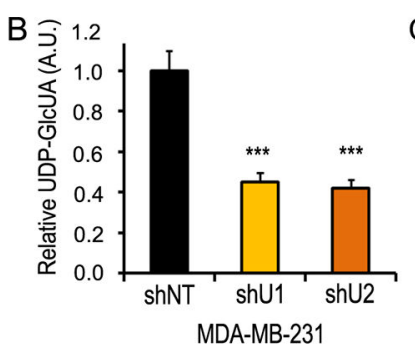

$\mathrm{F}$
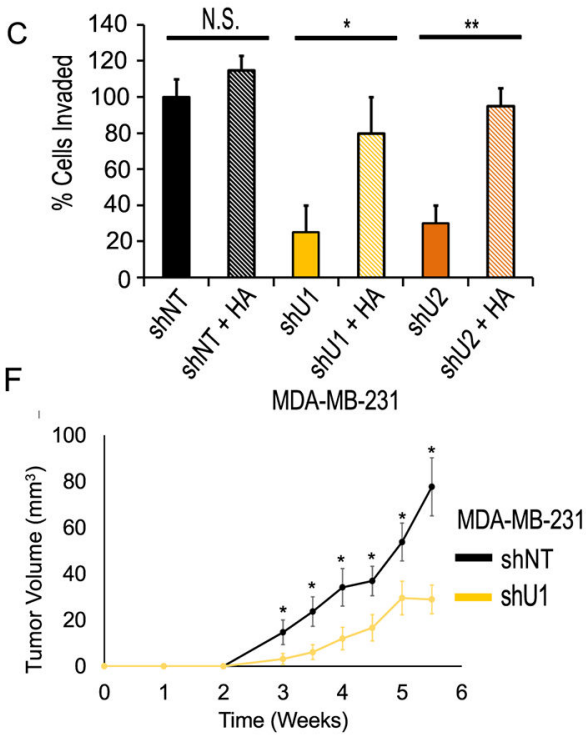

J

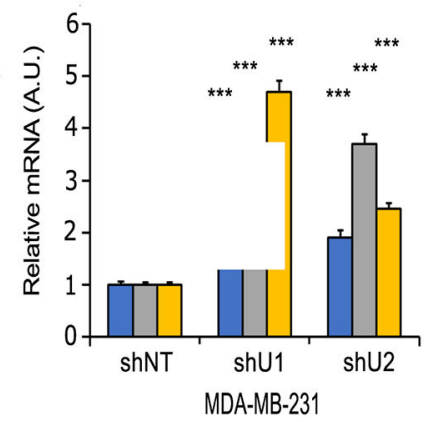

Differential Genes

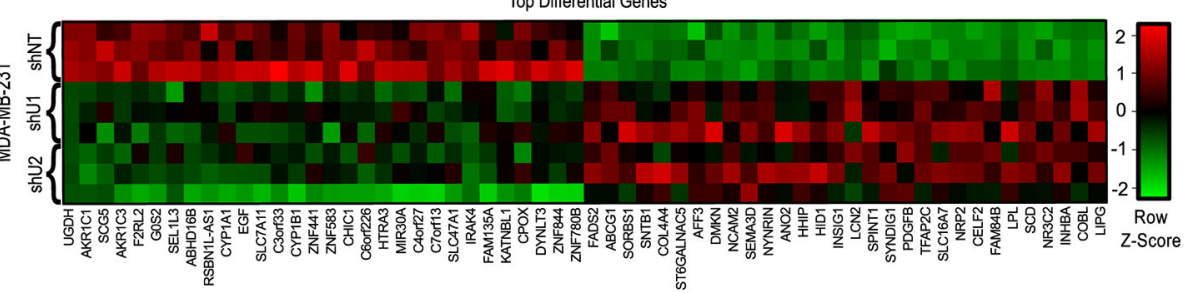

$\mathrm{K}$

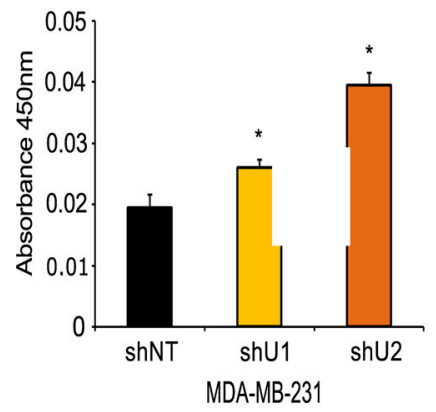

Figure 3: Effects of UGDH knockdown in a mesenchymal-like breast cancer cell line model. A) Western blot showing shRNA-mediated knockdown (KD) of UGDH in MDA-MB-231 (M231) by two independent shRNAs (shU1, shU2) compared to non-target shRNA (shNT).

B) UGDH KD reduces intracellular UDP-GlcUA. C) UGDH KD reduces cellular invasiveness in an HA-dependent manner as measured by transwell invasion assay. D) UGDH KD significantly reduces colony formation in soft agar. E) IHC of CAM models stained for UGDH (red), hyaluronic acid (Blue), with methyl green counter stain. 20X Magnification, scale bars are 50 $\mathrm{mm}$. F) UGDH KD tumors (shU1) in SCID-Beige mice grow at reduced rates compared to control shNT tumors $(\mathrm{n}=8)$. Two tumors from each cell line 
were removed once tumor volume of $50 \mathrm{~mm}^{3}$ was reached (4.5 weeks and 5.5 weeks post injection for shNT and shU1, respectively) to examine differences in HA levels in early stage tumors. (G) Pulmonary metastasis was reduced in UGDH KD cells compared to control shNT cells following tail-vein injection as quantified by luciferase signal. $\mathrm{H}$ ) Heatmap of top 60 differential genes with FDR-corrected p-value $<0.05$, genes are ordered from lowest to greatest fold-change in UGDH KD models vs. control. I) Network of pathways associated with overexpressed genes in UGDH KD models indicates enrichment of genes involved in lipid metabolism and PPAR signaling. J) RT-PCR confirmation that FADS2, LIPG, and LPL lipid metabolism genes are upregulated following UGDH KD. K) PPAR-gamma transcription factor binding assay shows UGDH knockdown increases PPARgamma DNA binding activity in MDA-MB-231 cells. N.S. $=$ not significant, $* \mathrm{p}<0.05, * * \mathrm{p}$ $<0.01, * * *<0.001$, versus control. 
A

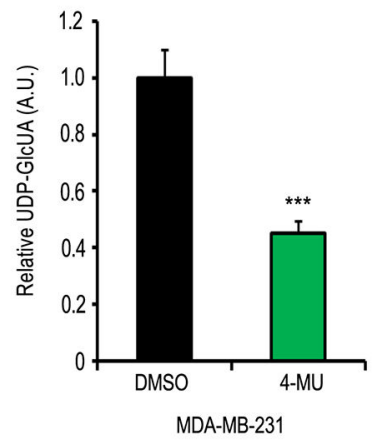

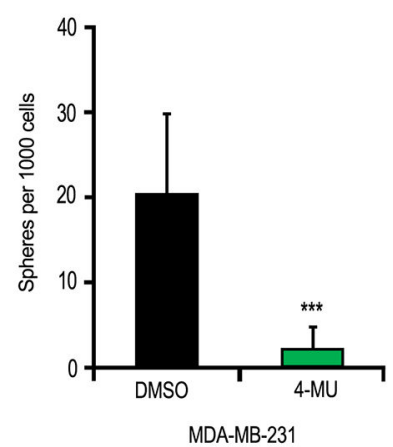

C

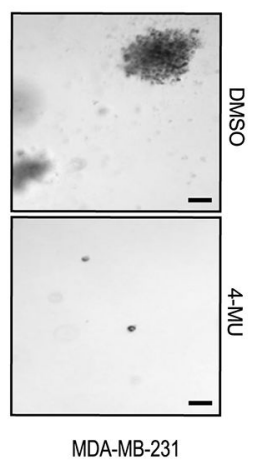

D

$\mathrm{E}$
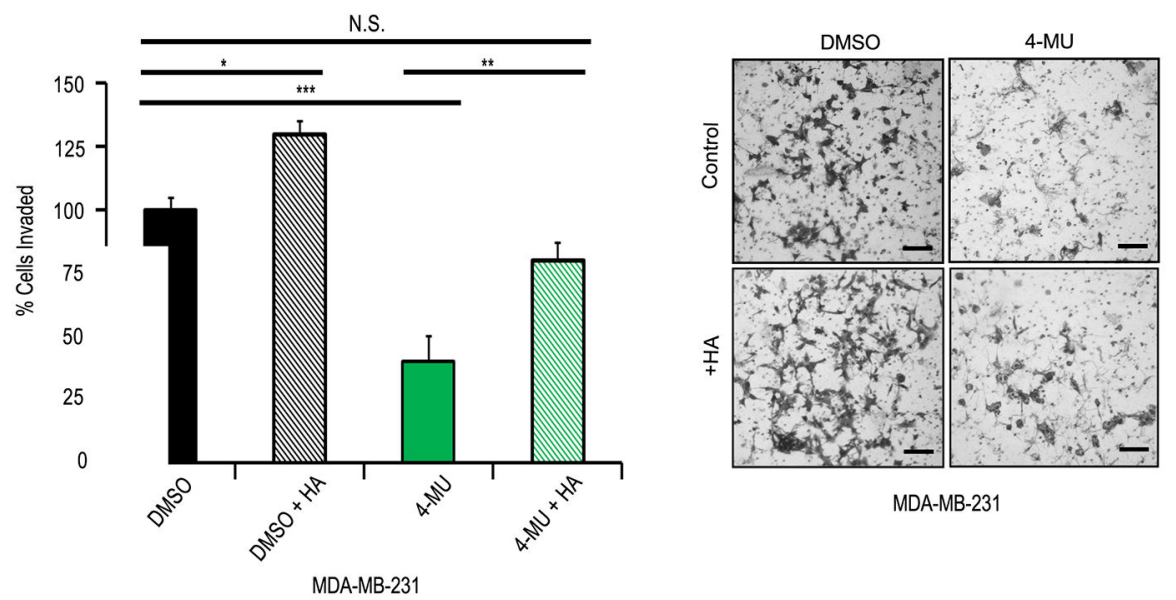

MDA-MB-231

G

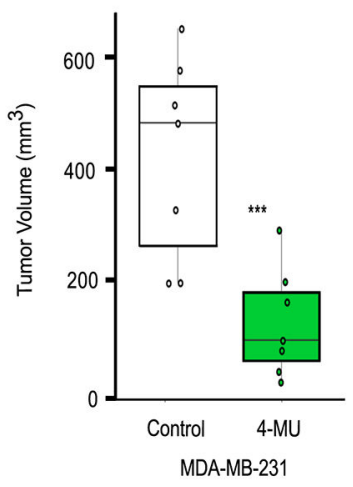

Figure 4: Effects of pharmacological UDP-glucuronic acid depletion in a mesenchymal-like breast cancer cell line model.

A) MDA-MB-231 cells treated with 1mM 4-methylumbelliferone (4-MU) had significantly decreased intracellular UDP-GlcUA. B) MDA-MB-231 cells treated with 1mM 4methylumbelliferone formed significantly fewer colonies in soft agar. C) Representative images from colony formation assay. D) Treatment with $1 \mathrm{mM} 4-\mathrm{MU}$ significantly reduced cellular invasiveness of MDA-MB-231 cells in a hyaluronic acid (HA) -dependent manner. E) Representative images from transwell invasion assay. F) Images of excised MDAMB-231 xenograft tumors from mice which were administered $225 \mathrm{mg} / \mathrm{kg} 4-\mathrm{MU}$ or $2 \%$ 
sucrose (Control) via daily oral gavage. G) Boxplots of excised tumor volumes show 4-MU treatment significant delayed tumor growth. Scale bars $=100 \mu \mathrm{m}$. N.S. $=$ not significant, $* \mathrm{p}$ $<0.05, * * \mathrm{p}<0.01, * * *<0.001$, versus control. 
Table 1.

Recurrence-free survival scores of mesenchymal-like metabolic genes.

\begin{tabular}{|c|c|c|c|}
\hline Gene Name & RFS HR $(\mathbf{9 5} \% \mathbf{C I})$ & Logrank P & Affy ID \\
\hline UGDH & $1.26(1.02-1.56)$ & 0.03 & 203343_at \\
\hline VKORC1 & $1.23(1-1.52)$ & 0.052 & 217949_s_at \\
\hline GNS & $1.14(0.93-1.41)$ & 0.21 & 212334_at \\
\hline ATP6V0D1 & $1.12(0.91-1.38)$ & 0.28 & 212041_at \\
\hline CSGALNACT2 & $1.1(0.89-1.36)$ & 0.36 & 222235_s_at \\
\hline PPAP2A & $1.1(0.89-1.36)$ & 0.38 & 210946_at \\
\hline MME & $1.08(0.87-1.33)$ & 0.49 & 203434_s_at \\
\hline GALNT10 & $1.01(0.82-1.25)$ & 0.9 & 212256_at \\
\hline MAN1A1 & $0.99(0.8-1.22)$ & 0.94 & 221760_at \\
\hline HAS2 & $0.94(0.76-1.16)$ & 0.58 & 206432_at \\
\hline PPAP2B & $0.7(0.56-0.86)$ & 0.00083 & 212230_at \\
\hline
\end{tabular}


Table 2.

List of primer sequences used for RT-PCR in this study.

\begin{tabular}{|c|c|}
\hline Primer & Sequence \\
\hline UGDH - Forward & CAACAGCGATTGGAATGGACC \\
\hline UGDH - Reverse & TCTGGCAAATTCAGAGCCTCA \\
\hline HAS2 - Forward & TCCTGGATCTCATTCCTCAGC \\
\hline HAS2 - Reverse & TGCACTGAACACACCCAAAATA \\
\hline FADS2 - Forward & TCAAGAACTTGCCCACGAAT \\
\hline FADS2 - Reverse & TGACCGCAAGGTTTACAACA \\
\hline LPL - Forward & TCAGCTGTGTCTTCAGGGG \\
\hline LPL - Reverse & CTCCAGAGTCTGACCGCCT \\
\hline LIPG - Forward & GTCAACAAAGAGGTGGACGG \\
\hline LIPG - Reverse & GCTGTGGACTCAACGATGTC \\
\hline GAPDH - Forward & GGAGCGAGATCCCTCCAAAAT \\
\hline GAPDH - Reverse & GGCTGTTGTCATACTTCTCATGG \\
\hline
\end{tabular}


Table 3.

List of MRM transitions used for glucose flux studies.

\begin{tabular}{|l|c|c|l|}
\hline \multicolumn{1}{|c|}{ Compound Name } & Precursor Ion & Product Ion & Polarity \\
\hline Glucose $\mathrm{m}+0$ & 179 & 59 & Negative \\
\hline Glucose $\mathrm{m}+6$ & 185.0762 & 61 & Negative \\
\hline UDP-glucuronic acid $\mathrm{m}+0$ & 579.02 & 402.9 & Negative \\
\hline UDP-glucuronic acid $\mathrm{m}+6$ & 585.02 & 402.9 & Negative \\
\hline UDP-N-Acetylglucosamine $\mathrm{m}+0$ & 606 & $282 / 385 / 79$ & Negative \\
\hline UDP-N-Acetylglucosamine $\mathrm{m}+6$ & 612 & $288 / 79$ & Negative \\
\hline Citrate $\mathrm{m}+0$ & 191.0 & $87 / 111$ & Negative \\
\hline Citrate $\mathrm{m}+2$ & 193.0 & 113 & Negative \\
\hline Citrate $\mathrm{m}+4$ & 195.0 & 114 & Negative \\
\hline
\end{tabular}


Table 4.

List of components for human-specific Alu sequence RT-PCR quantification.

\begin{tabular}{|c|c|}
\hline Component & Sequence \\
\hline YB8-ALU-S68 primer & GTCAGGAGATCGAGACCATCCT \\
\hline YB8-ALU-AS244 primer & AGTGGCGCAATCTCGGC \\
\hline YB8-ALU-167 probe & 6-FAM-AGCTACTCGGGAGGCTGAGGCAGGA-TAMRA \\
\hline
\end{tabular}

